\title{
Finite element analysis of thermoelastic contact problem in functionally graded axisymmetric brake disks
}

\begin{abstract}
An analysis of thermoelastic contact problem of functionally graded (FG) rotating brake disk with heat source due to contact friction is presented. Finite element method (FEM) is used. The material properties of disk are assumed to be represented by power-law distributions in the radial direction. The inner and outer surfaces considered are metal and ceramic, respectively. Pure material is considered for the brake pad. Coulomb contact friction is assumed as the heat source. It is divided into two equal parts between pad and brake disk which leads to thermal stresses. Mechanical response of FG disks are compared and verified with the known results from the literatures. The results show that the maximum value of radial displacement in mounted FG brake disk is not at outer surface. It is found that the all areas between pad and brake disk is in full-contact status when the ratio of pad thickness to brake disk thickness is 0.66 . It is observed that the total strain due to thermomechanical load is negative for some parts of the disks, whereas, the thermal strains are always positive. It can be concluded that gradation index of the metal-ceramic has significant effect in the thermomechanical response of FG disks.
\end{abstract}

Keyword: Functionally graded material; Brake disk; Finite element method; Thermoelastic 